\title{
Methodology for the Evaluation of Drugs in Pregnant Women
}

\author{
Marina Chauvenet, ${ }_{1}^{1}$ Alain Rimailho, ${ }^{2}$ Natalie Hoog-Labouret ${ }^{1}$ and the Participants in \\ Round Table No. 5, Giens XVIII**
}

1 Agence Française de Sécurité Sanitaire des Produits de Santé, Saint-Denis, France

2 Schering-Plough, Levallois-Perret, France

\begin{abstract}
Although drugs are prescribed during pregnancy with some reluctance, they fulfil a real need in some circumstances. Adequate drug evaluation is thus essential, either based on efficacy and safety or mainly safety, using available data from non-pregnant women. Evaluation methodology is not fundamentally different during pregnancy. Recommendations for drug development are formulated on the basis of the most common situations as well as specific suggestions, thus raising the awareness of the different partners participating in healthcare (institutions, the pharmaceutical industry and prescribers). In particular, regulatory and economic incentives superimposed upon those recommendations adopted in Europe and the US for orphan diseases should be put into place to assist in the evaluation of drugs used in obstetrics. Medical needs in obstetrics should be better identified, and labelling of drugs for use during pregnancy should be better directed towards prescribers; a national registry of pregnancies should be established in France.
\end{abstract}

Keywords: drug, pregnancy, evaluation, regulatory incentives.

Text received May 6, 2003; accepted May 26, 2003

\section{Background}

Given that almost $35 \%$ of women take some kind of medication other than iron and vitamin supplements during pregnancy, prescription and drug consumption during pregnancy represent an inescapable reality. ${ }^{[1,2]}$

Currently, in France, the legal notices for drugs that are likely to be prescribed during pregnancy all focus on the risks of the drug to the unborn baby, and are grouped according to three types of potential effects: teratogenic (producing malformations, linked to exposure at the beginning of pregnancy); fetotoxic (with a risk commencing at the second trimester of pregnancy); and neonatal (most frequently linked to exposure at the end of the pregnancy or during birth).

The fundamental evaluation of drug risks in pregnant women that were used when drawing up these recommendations were the subject of a previous round table. ${ }^{[3]}$ Therefore, they will not be discussed again during this overview of evaluation methodology for drugs used in pregnant women.

Thus, health professionals who may need to prescribe drugs to pregnant women will now find information notices about the risks to the unborn baby in the section entitled Pregnancy and Breastfeeding of the French Marketing Authorisation (AMM) for all drugs; however, they will also notice that drugs with a specific indication for use during pregnancy are extremely rare and that information on the dosage to be prescribed throughout pregnancy is almost completely lacking. This is surprising considering the important pharmacokinetic and sometimes dynamic modifications induced by the physiological changes in pregnancy, particularly during the third trimester. ${ }^{[4]}$

$\dagger$ For a list of participants, please see the end of the article.

* Articles, analyses and proposals arising from the Giens Clinical Pharmacology Meetings are the responsibility of their authors and do not reflect the views held by their supervisory organisation. 
It is possible to believe that this situation is connected to an almost complete lack of evaluation of drugs used in pregnant women, the logical conclusion being that it is impossible to propose validated rules for use.

Consequently, although anti-emetic drugs are prescribed to treat the nausea and/or vomiting, a frequent occurrence during the first trimester, there is no clear demonstration of their effectiveness, particularly in comparison with non-pharmacological measures. ${ }^{[5]}$ Similarly, although spiramycin is the reference preventive treatment in France for reducing mother-to-baby transmission of toxoplasmosis in seropositive mothers, there is insufficient evaluation of its real effectiveness. ${ }^{[6]}$

It is clear that clinical drug development in pregnant women is a sensitive area for three reasons:

- the legitimate ethical reason - this carries considerable weight even though, since the thalidomide affair, some irrational fears are also involved;

- the over cautiousness of the pharmaceutical industry, which is associated with medico-legal problems and small financial rewards;

- and the lack of regulatory requirements to produce information about the use of the drug in pregnant women for registration.

However, there are some not insignificant initiatives that demonstrate the value and feasibility of dealing systematically with the question of the methodology of evaluating drugs for use in pregnant women.

Interestingly, the Cochrane methodology, which is based on the meta-analysis approach, was applied to obstetrics in the early days; thus, there are a number of drug studies found in this source. ${ }^{[7]}$

\section{Ethical and Regulatory Situation}

Before identifying the clinical situations where drug development specific to pregnant women seems desirable - indispensable even - and before detailing the available tools, we summarise the present ethical and regulatory situation.

In France, the law for the protection of people taking part in biomedical research, called the 'Huriet-Sérusclat' law, ${ }^{[8]}$ has provisions for performing clinical trials during pregnancy with or without direct individual benefit (DIB). It defines specific conditions for performing research without DIB; this can only be done "if it does not present any serious foreseeable risk to the subject's health or the health of their child, if it contributes to knowledge about the characteristics of pregnancy or childbirth, and if it cannot be achieved in other ways". The new European
Directive ${ }^{[9]}$ on clinical trials, however, does not make any specific mention of pregnancy.

The information supplied by the AMM in France under the heading Pregnancy and Breastfeeding is the result of an evaluation of all the available data (preclinical and clinical), by therapeutic class, on the risks and benefits during pregnancy, drawn up by a multidisciplinary working group from the AMM Commission. This process aims to produce a practical guide for the prescriber by taking into account the medical requirements.

Current European regulations include an obligation for pharmaceutical companies to declare any pregnancies reported during exposure to one of their medications, particularly if there are any abnormalities during pregnancy or during the neonatal period, and especially if any malformation occurs. ${ }^{[10]}$ But the evaluation of drugs administered during pregnancy is increasingly being structured at the European level: guidelines intended to reinforce obligations for post-AMM monitoring of pregnancies exposed to a priority list of drugs are currently being drawn up, and there is a working group with the responsibility of harmonising the methods of evaluation and information on pregnancy in the Summary of Product Characteristics.

Finally, it should also be noted that the US Food and Drug Administration has begun to consider the question of clinical evaluation of drugs administered during the second and third trimesters of pregnancy, and the requirements for registers of pregnant women.

It would seem clear that clinical trials could fall within the development-plan parameters for a drug by including pregnant women.

In France, 25 clinical trials evaluating a drug used in pregnant women were registered with the French agency for the sanitary safety of health products (Afssaps [Agence française de sécurité sanitaire des produits de santé]) for the period January 1998 to September 2002. Most of these trials were classed as with DIB, and only nine were sponsored by a pharmaceutical firm.

This must obviously be considered in parallel with the overall number of clinical trials, approximately 1500, with about 100 of these involving children, registered each year.

It was considered that an analysis of the recent past would be useful to identify which elements have been used or would be possibilities in suggesting legal requirements for products developed for, or used, during pregnancy. A good model for this is atosiban, which has recently been registered for purely obstetric indications, after a complete development process, by centralised European procedures. In contrast, nifedipine is sometimes used for the same indication (risk of preterm labour [PTL]) even though the indication is not given in the AMM, since this drug had demonstrated efficacy versus $\beta$-agonists together with 
fewer maternal adverse effects; ${ }^{[11]}$ however, there are still some uncertainties about neonatal safety with regard to rare, but potentially serious, adverse effects. ${ }^{[12]}$ In an effort to ease the legal requirements for some low molecular weight heparins with medical indications in pregnancy, Afssaps, in consultation with the pharmaceutical industry, has taken steps towards evaluating fetal risk by studying drug transfer across the placenta.

\section{Mother/Baby Pharmacokinetics}

Relevant information on the pharmacokinetics of the drug, in particular the degree of drug transfer across the placenta, is absolutely vital.

Pregnancy alters pharmacokinetic parameters by delaying gastric emptying time, increasing intestinal transit time, increasing blood volume, and increasing the glomerular filtration rate. These changes can affect the absorption, bioavailability and elimination of numerous drugs.

Passage across the placenta mainly occurs as passive diffusion; this depends on the molecular characteristics of the drug (lipid solubility, molecular weight, $\mathrm{pH}$, protein-binding characteristics, and enzyme activity in the placenta). ${ }^{[13]}$

In practice, it can be assumed that, with the exception of very high molecular weight molecules such as insulin, almost all drugs pass across the placenta. Therefore, when attempting to define the amount of drug that passes across the placenta, the analysis should include information from animal models, ex-vivo human data (placenta perfusion technique), and in-vivo human data comparing mother/fetus circulating levels using cord blood sampled at birth.

Nevertheless, all these methods give only a partial picture of the physiological situation. Levels measured in cord blood at the time of birth provide only a snapshot and do not take kinetics into account; to determine these, a relatively large number of samples need to be taken from the mother-umbilical cord unit. Ex-vivo techniques provide a dynamic approach, although they can be difficult to perform, since they require specific expertise and careful quality control. They are particularly useful for comparing differentiated passage across the placenta of products in the same drug class. ${ }^{[14]}$ The in-vivo and ex-vivo results for drugs that cross the placenta by passive diffusion are very similar. ${ }^{[15]}$ On the other hand, there are instances where the two approaches have shown contradictory results, such as those found with the protease inhibitor class of antiretrovirals for which initial in-vivo studies suggested that they did not cross the placenta, ${ }^{[16]}$ but $e x$-vivo studies gave varied results depending on the molecule used. ${ }^{[17,18]}$

By combining all the theoretical data on the drug, and human and animal data obtained during the early development phases (in particular pharmacokinetics), a qualified (rather than a precisely quantified) estimation of the therapeutic range can be obtained from the clinical development of a new product, for both the mother and the fetus. With the prerequisite data on the ratio of fetal to maternal drug concentrations in the blood at birth, a pharmacokinetic profile in the mother, and data obtained ex vivo, sufficient information should be available to embark on an overall clinical evaluation of the product. This will be in addition to the requirements of the 1997 International Conference on Harmonisation (ICH) directive, which states that prior to the inclusion of pregnant women in clinical trials, all the reproduction toxicity studies and the standard battery of genotoxicity tests should be conducted. ${ }^{[19]}$

\section{The Different Pathological Situations}

The different pathological situations fall into four groups.

1. Exclusively fetal diseases: there is a low incidence of these; drug treatments and examples are as follows: spiramycin and pyrimethamine/sulfadiazine for toxoplasmosis, antiarrhythmics for fetal supraventricular tachycardia, L-thyroxin for in utero hypothyroidism, etc.).

2. Diseases occurring only during pregnancy: by definition the indications apply only to gravid women. These involve mainly drugs intended to induce labour or tocolytics (atosiban for example).

Once the prerequisites described in the previous section are fulfilled, it is possible to plan one or more randomised, placeboor reference product (if existing)-controlled clinical trials to demonstrate efficacy. By definition, these trials can be performed only in pregnant women. The principal criteria can be dictated by a concern to keep the patient numbers low, which means that the validity of any possible surrogate endpoints must be carefully considered, particularly if a dose-research phase seems unavoidable before proceeding to the proposed phase III study.

A systematic follow-up study of the babies should be planned and organised, and should be continued well beyond the neonatal period.

When this development phase has been concluded, a complete obstetric indication should be possible for the product studied if a positive risk to benefit ratio is established with a study population large enough to produce efficacy and safety analyses for both mother and baby.

3. Diseases occurring in pregnancy that could be treated with a drug that has already been tested and validated in humans: the drug may be beneficial for the pregnant woman and also the fetus; this is the situation with transmissible infectious diseases such as herpes or AIDS. ${ }^{[20,21]}$ The requirement here is to appre- 
ciate the true medical needs of the mother and child, i.e. to know how to manage the risk associated with undertaking clinical trials that aim to show product efficacy early in the life of the product; this has been the case in the extremely urgent situation of the AIDS epidemic, where the mother-to-baby transmission rates are $20-25 \%$. This type of fast-track trial is possible as soon as the pharmacokinetic prerequisites have been established; it will be combined with the collection of spontaneous pharmacovigilance reports, including reports of cases of pregnancy occurring during the time the study product is being prescribed, and data from any cohorts. A comparative, randomised standard clinical trial designed to show efficacy can be performed, using either a reference product as the control or placebo if there is no reference product, or if the reference product has not been sufficiently evaluated in pregnant women in this situation. Mention should be made here of the value and validity of a surrogate endpoint; this could become necessary if the study population to be included in the trial becomes too large as a result of the choice of the main criteria and the low incidence of events that are to be controlled by the product.

Normally a follow-up of the children should be planned and carried out, but this can cause major feasibility problems, mainly due to cost.

If a favourable risk to benefit ratio has been established by the end of this process, a specific product indication for pregnant women should have been obtained.

Thus, although in categories two and three described above, the process is seeking to demonstrate efficacy as well as collecting safety data, and may ideally produce an obstetric indication, in category four described below safety evaluations take precedence and the conclusions should lead to an appropriate modification to drug information (recommendation).

4. Pre-existing chronic diseases in pregnancy, where treatment must be continued throughout the pregnancy (e.g. epilepsy, asthma or depression): in this situation, two questions need to be addressed:

- does the pregnancy alter physiopathology and therefore possibly alter the progression of the disease?

- does the pregnancy alter the pharmacological characteristics of a given medication?

A negative response to these two questions, based on reasoned arguments and using current pharmacovigilance data, should indicate that prescribing information and guidelines can be added to the legal specifications; this could even include dosage suggestions for use in pregnancy.

Conversely, a positive response to one of these questions should be an incentive to perform a pharmacokinetic profile of the product concerned, if only for the third trimester, so that dos- age guidelines and information for the prescriber can be added to the legal specifications, also taking into account current pharmacovigilance data. This should assuage the frequently expressed ethical concerns ${ }^{[22]}$ that result in the complete absence of any mention of dosage guidelines for a drug in pregnancy. Physiological changes can induce profound alterations to the pharmacokinetics of a drug and can make a product ineffective by reducing concentrations over several weeks or months, even though the normal dosage has been given during pregnancy. ${ }^{[23]}$ This type of pharmacokinetic study is even more vital if the drug has a narrow therapeutic range and a correlation has been established between the dose and the serum concentrations on one hand, and the dose and the pharmacological effect on the other hand in patients who are not pregnant.

\section{The Pragmatic Approach}

Besides this standard approach, the possibility of adopting a more pragmatic attitude in certain cases was discussed. In fact, several molecules have been the subject of one or more efficacy trials without there having been prior phase I/II studies. The quality of some of these trials has been good enough for them to have been included in meta-analyses (calcium channel blockers to inhibit PTL or misoprostol for inducing labour, for example). In addition, there is sometimes considerable pharmacovigilance information, and therefore information about the safety for use, available from the drug monitoring records (e.g. acyclovir).

Would not it be possible, particularly where measures taken in practice are validated by recommendations drawn up at consensus conferences, to envisage regulatory validation enabling a substantial modification of the information for the prescriber? This proposal was suggested for well established products that have been sold as generics for a long time, and for which it is very difficult to imagine that it would be possible to interest the pharmaceutical companies in drug development plans specifically for pregnant women. The round table drew up a list of suggested drugs (table I).

\section{Action Points}

\section{1 Policies}

The role of the institutions seems crucial if we wish to construct and develop a really effective evaluation procedure for drugs for use in obstetrics.

\subsubsection{Regulatory Proposals at the European Level}

The proposition that the 'orphan diseases' approach should be applied to diseases specific to pregnancy, with certain prod- 
Table I. Proposition for drugs frequently used in the practice of obstetrics

\begin{tabular}{ll}
\hline Product (INN) & Indications \\
\hline Nifedipine & PTL \\
Nicardipine & PTL \\
Misoprostol & Inducing labour \\
Acyclovir & Prevention of recurrent genital herpes \\
Amoxicillin & Premature membrane rupture \\
Chenodeoxycholic acid & Cholestasis during pregnancy \\
Trinitrine & Emergency tocolytic \\
\hline INN = international nonproprietary name; PTL = preterm labour.
\end{tabular}

ucts claiming only that indication, was passed unanimously. This measure would be aimed at the pharmaceutical industry and maybe also at the institutions that are in a position to sponsor clinical trials. Regulatory and financial incentives, superimposable on those adopted by Europe ${ }^{[24]}$ and the US ${ }^{[25]}$ for orphan medicines, would energise research efforts in the area of development of drugs specifically suitable for pregnant women. In addition, given that the status of the fetus and that of the premature or full-term newborn are inextricably linked, it seems rational to expect that the situation of the pregnant woman is at least taken into account in future European paediatric legislation.

\subsubsection{National Epidemiology Proposal}

The clinicians and epidemiologists in the group considered that, after years of 'gestation' and successful experience in one French department, ${ }^{[26]}$ a national register of pregnancy outcomes or 'pregnancy outcome certificates' should finally be organised in France. This register could be used to analyse various types of information, in particular drug exposure, with systematic collection of, for example, various exposures over limited periods. Failing that, the value of computerising the obstetric records at the regional level was emphasised.

\subsection{Regulatory Affairs}

\subsection{Information Supplied by the Marketing Authorisation}

A pragmatic initiative suggested by the Afssaps representatives was that it would be possible to carry out a rapid revision of the information given under the 'Pregnancy' heading for a list of 'priority' drugs (in terms of obstetric requirements) that have had 'some' validated assessment in the literature. This list (table I), proposed by the obstetricians at the round-table, would need to be discussed jointly with the scientific bodies.

This step, which is similar to the Afssaps initiatives in the area of paediatric medication, would be aimed at reducing the still considerable gap between the information in the AMM and what happens in practice, at least for essential drugs. The similar, but not identical, problem of breastfeeding also requires more attention. Information on drug products that could be validated according to the current template (July 2002) of the Ad Hoc group at Afssaps could be given a star system in the Dictionnaire des Spécialités Vidal, so that the prescribers could find them more easily, particularly when there is lack of harmonisation in labelling, which is caused by European registration procedures compared with national evaluation. This information should also be available on the Afssaps website.

Still on the subject of harmonisation, Afssaps should be involved in drawing up the next editions of the various European guidelines, so that the French experience can be used effectively.

\subsubsection{Revision of the Huriet-Sérusclat Law}

When national legislation is revised to come into line with the new European Directive on clinical trials, the present distinction between trials with or without DIB should be maintained at the very least. The group also proposes following the example of the requirements for paediatric drugs, and make a point of mentioning the requirement for a member of the Ethics Committee to be competent in obstetrics and neonatal care, so that the Committees are qualified to pronounce on the validity of obstetric protocols.

\subsection{Ethics}

There should be some consideration of ethical aspects jointly with the French National Ethics Committee and the various other people involved, as the strongest reservations will not necessarily be raised by women or their partners, nor by the clinicians, but by the industrial partners or the institutions.

\section{Conclusion}

Clinical development of drugs for use during pregnancy will encounter all types of well identified problems, particularly as there is a lack of obligation on the part of the pharmaceutical industry to supply data from pregnant women when applying for authorisation, other than for obviously targeted obstetric indications. It seems that a serious effort of national and European political will is needed if we are to make any real progress in our knowledge and use of drugs in pregnant women. This means that drugs used to treat only conditions associated with pregnancy should be incorporated into the recent European regulations on orphan drugs and that a national register of pregnancies in France should be organised, similar to the Swedish register, for example. Furthermore, there must be a radical improvement in the definition of priority medical requirements, and available data must be used to make proposals for adapting the information for the prescriber in the AMM. These proposals should improve the present 
unsatisfactory situation. Although it is right that the evaluation of risk to the unborn child should remain at the centre of everyone's concerns, this should not obscure the fact that there is also a requirement to clarify the process of evaluation of the benefits and risks to the mother and the benefit to the child.

\section{Participants}

F. Audibert (Hôpital Antoine Béclère, Clamart), F. Bavoux (Hôpital Saint-Vincent de Paul, Paris), M.J. Boutroy (CHU, Nancy), D. Brasseur (EMEA, CPMP, London), P. Carlier (Hôpital Fernand Widal, Paris), E. Elefant (CHU St-Antoine, CRAT, Paris), M. Gersberg (Sanofi-Synthelabo, Le Plessis-Robinson), F. Goffinet (Maternité Port-Royal, Paris), E. JacqzAigrain (Hôpital Robert Debré, Paris), D. Lemery (Maternité de l'Hôtel-Dieu, Clermont-Ferrand), L. Mandelbrot (CHU Cochin, Paris), V. Mirelesse (Institut de Puériculture et Périnatalogie, Paris), B. Saint-Salvi (Afssaps, St-Denis), C. Tchinou (Afssaps, St-Denis), T. Vial (Centre Hospitalier E.Herriot, Lyon).

\section{References}

1. Lacroix I, Damase-Michel M, Lapeyre-Mestre M, et al. Prescription of drugs during pregnancy in France. Lancet 2000; 356 (9243): 1735-6

2. McElhatton P. Pregnant women and drug safety. Good Clin Pract J 2002; 9: 15-7

3. Eléfant E, Sainte-Croix A, et les membres de la table ronde $n^{\circ} 6$ de Giens XII. Evaluation du risque médicamenteux chez la femme enceinte : méthodologie d'évaluation et gestion du risque. Thérapie 1997; 52: 307-11

4. Loebstein R, Lalkin A, Koren G. Pharmacokinetic changes during pregnancy and their clinical relevance. Clin Pharmacokinet 1997; 33: 328-43

5. Mazzotta P, Magee LA. A risk-benefit assessment of pharmacological and nonpharmacological treatments for nausea and vomiting of pregnancy. Drugs 2000; 59: 781-800

6. Wallon M, Liou C, Peyron F. Congenital toxoplasmosis: systematic review of evidence of efficacy of treatment in pregnancy. BMJ 1999; 318: 1511-4

7. Anonymous. Stratégies : menaces d'accouchement prématuré. Prescrire 2002; 22: 676-686

8. Loi pour la protection des personnes qui se prêtent à des recherches biomédicales. Code de la Santé Publique. Livre II bis : article L209-4

9. Directive 2001/20/EC of the European Parliament and of the Council of 4 April 2001 on the approximation of the laws, regulations and administrative provisions of the Member States relating to the implementation of good clinical practice in the conduct of clinical trials on medicinal products for human use. Official Journal of the European Communities, L121-1/05/2001. Available from URL: http://europa.eu.int/eur-lex/pri/en/oj/dat/2001/1_121/1_12120010501 en00340044.pdf

10. European Agency for the Evaluation of Medicinal Products (EMEA). Notice to marketing authorization holders: pharmacovigilance guidelines [online].
CPMP/PhVWP/108/99, 1999 Jan 29. Available from URL: http://www.tga. health.gov.au/docs/pdf/euguide/phvwp/010899en.pdf

11. Tsatsaris V, Papatsonis D, Goffinet F, et al. Tocolysis with nifedipine or beta-adrenergic agonists: a meta-analysis. Obstet Gynecol 2001; 97: 840-7

12. Rosen LJ. The great tocolytic debate: some pitfalls in the study of safety. Am $\mathbf{J}$ Obstet Gynecol 2001; 184: 1-7

13. Gablot D, Laurichesse H, Lemery D. Prévention des risques fœtaux. Rev Prat 2002; 52: 751-64

14. Bourget P, Roulot C, Fernandez H. Transfert transplacentaire et métabolisme fœtoplacentaire des médicaments : méthodes d'études, apports et implications thérapeutiques. Thérapie 1994; 49: 481-97

15. Tuntland T, Odinecs A, Pereira CM, et al. In vitro models to predict the in vivo mechanism, rate, and extent of placental transfer of dideoxynucleoside drugs against human immunodeficiency virus. Am J Obstet Gynecol 1999; 180: 198206

16. Mirochnick M, Dorenbaum A, Holland D, et al. Concentrations of protease inhibitors in cord blood after in utero exposure. Pediatr Infect Dis J 2002; 21: 835-8

17. Forestier F, de Renty P, Peytavin G, et al. Maternal-fetal transfer of saquinavir studied in the ex vivo placental perfusion model. Am J Obstet Gynecol 2001; 185: $178-81$

18. Casey BM, Bawdon RE. Placental transfer of ritonavir with zidovudine in the ex vivo placental perfusion model. Am J Obstet Gynecol 1998; 179: 758-61

19. International Conference on Harmonisation of Technical Requirements for Registration of Pharmaceuticals for Human Use (ICH). Therapeutic Products Directorate Guideline ICH. Harmonized tripartite guideline (M3): timing of non-clinical safety studies for the conduct of human clinical trials for pharmaceuticals, 1997 Jul

20. Mandelbrot L. Syndrome d'immunodéficience acquise et grossesse. Encycl Méd Chir (Traité d'Obstétrique) $2001: 5-039 D-40$

21. Mandelbrot L. Vertical transmissions of viral infections. Curr Opin Obstet Gynecol 1998; 10: 123-8

22. Wood SF, Miller MA, Hollinger KA. Why women should be included in clinical trials? Good Clin Pract J 2002 Sep; 9: 3-4

23. Gérard-Coué MJ, Bréart G, Spriet A. Les femmes dans les essais cliniques (Giens X). Thérapie 1995; 50: 363-8

24. Règlement $141 / 2000$ du parlement européen et du conseil du 16 décembre. Journal Officiel des Communités Européennes 2000 ; nº018 du 22 janvier 2000 : 0001-0005

25. Orphan drug regulations: final rule. Federal Register 62076 V.57, 1992

26. Papiernik E, Bucourt M, Zeitlin J, et al. Evolution de la régionalisation des soins périnatals dans le département de la Seine-Saint-Denis de 1989 à 1999. J Gynécol Obstét Biol Reprod (Paris) 2001; 30: 338-43

Correspondence and offprints: Alain Rimailho, Schering-Plough, 92 Rue Baudin, 92307 Levallois-Perret Cedex, France.

E-mail: alain.rimailho@spcorp.com 